\title{
GEOMETRÍA DE LAS FOLIACIONES LINEALES EN $\mathbb{C}^{2}$
}

\section{Renato Benazic Tomé*}

Resumen: En el presente trabajo, estudiamos el comportamiento geométrico de las soluciones de una ecuación diferencial ordinaria compleja bidimensional. Probamos que la geometría de las hojas depende de la posición que ocupan los autovalores en el plano complejo y daremos la descripción completa de esta geometría.

Palabras clave: Foliaciones analíticas complejas. Foliaciones por curvas. Ecuaciones Ordinarias Complejas.

\section{GEOMETRY OF THE LINEAR FOLIATIONS IN $\mathbb{C}^{2}$}

Abstract: In this work, we study the geometric behavior of the solutions of a bidimentional complex ordinary diferential equation. We prove that the geometry of the leaves depend on the.position of the eigenvalues in the complex plain and we will give a completely description of these geometry.

Key words: Complex Analitic Foliations. Foliations by curves. Complex Ordinary Equations.

\section{Introducción}

A lo largo de todo el artículo, $\mathbb{C}^{2 \times 2}$ denotará al conjunto de las matrices cuadradas $2 \times 2$ cuyas entradas son números complejos.

Dada la matriz $A=\left[\begin{array}{ll}a_{11} & a_{12} \\ a_{21} & a_{22}\end{array}\right] \in \mathbb{C}^{2 \times 2}$ y el par $z_{0}=\left(z_{1}^{0}, z_{2}^{0}\right) \in \mathbb{C}^{2}$, podemos considerar su Problema de Valores Iniciales (PVI) Lineal

$$
\mid \begin{aligned}
& z_{1}^{\prime}=a_{11} z_{1}+a_{12} z_{2}, \quad z_{1}^{\prime}(0)=z_{1}^{0} \\
& z_{2}^{\prime}=a_{21} z_{1}+a_{22} z_{2}, \quad z_{2}^{\prime}(0)=z_{2}^{0}
\end{aligned}
$$

o equivalentemente

$$
\mid \begin{array}{ll}
z^{\prime} & =A z \\
z(0) & =z_{0}
\end{array}
$$

Es bien conocido (ver [1], [2]) que la única solución del P.V.I. (1) viene dada por

$$
\begin{aligned}
\varphi: \mathbb{C} & \rightarrow \mathbb{C}^{n} \\
T & \mapsto \varphi(T)=e^{T A} z_{0} .
\end{aligned}
$$

en donde $e^{T A}=\sum_{j=0}^{\infty} \frac{T^{j}}{j !} A^{j}$, es la exponencial de la matriz $T A$.

*Universidad Nacional Mayor de San Marcos - Facultad de Ciencias Matemáticas, Instituto de Matemática y Ciencias Afines - IMCA.

e-mail: benazic@imca.edu.pe 
El flujo $\varphi_{A}: \mathbb{C} \times \mathbb{C}^{2} \rightarrow \mathbb{C}^{2}$ asociado al campo lineal $A$, se define como

$$
\varphi_{A}(T, z)=e^{T A} z
$$

Este flujo complejo es una función holomorfa en $\mathbb{C} \times \mathbb{C}^{2}$ y satisface las siguientes condiciones:

$$
\begin{aligned}
& \text { 1. } \frac{\partial \varphi_{A}}{\partial T}(T, z)=A \cdot \varphi_{A}(T, z), \forall(T, z) \in \mathbb{C} \times \mathbb{C}^{2} . \\
& \text { 2. } \varphi_{A}(0, z)=z, \forall z \in \mathbb{C}^{2} . \\
& \text { 3. } \varphi_{A}\left(T_{1}+T_{2}, z\right)=\varphi_{A}\left(T_{1}, \varphi_{A}\left(T_{2}, z\right)\right), \forall T_{1}, T_{2} \in \mathbb{C}, \forall z \in \mathbb{C}^{2} .
\end{aligned}
$$

La importancia del concepto de flujo que acabamos de dar estriba en que él contiene toda la información cualitativa de las soluciones de una E.D.O. lineal.

A continuación, definimos el bien conocido concepto de conjugación entre matrices (ver [10], [5]):

Sean $A, B \in \mathbb{C}^{2 \times 2}$ y consideremos sus flujos asociados $\varphi_{A}$ y $\varphi_{B}$. Decimos que las matrices $A$ y $B$ son topológicamente conjugadas, lo que denotamos $A \equiv_{\text {top }} B$ si y sólo si existe un homeomorfismo $h: \mathbb{C}^{2} \rightarrow \mathbb{C}^{2}$ llamado conjugación topológica tal que

$$
h\left(\varphi_{A}(T, z)\right)=\varphi_{B}(T, h(z)), \quad \forall T \in \mathbb{C}, \forall z \in \mathbb{C}^{2}
$$

En el caso que $h$ sea un biholomorfismo, entonces decimos que $A$ y $B$ son analíticamente conjugadas y $h$ es llamado conjugación analítica. Por último, si $h$ es un isomorfismo lineal, entonces $A$ y $B$ son linealmente conjugados y en este caso $h$ es llamado conjugación lineal. Usaremos la notación $A \equiv_{\text {ana }} B$, (respectivamente $A \equiv_{\text {lin }} B$ ), para decir que $A$ y $B$ son analíticamente conjugados (respectivamente linealmente conjugados).

La importancia del concepto anterior se debe a que si dos matrices son conjugadas entonces las soluciones de sus PVI asociados tienen comportamiento geométrico similar. El siguiente resultado, cuya demostración puede ser encontrada en [1], nos dice esencialmente que las matrices cuadradas de orden $n$ sólo admiten dos clasificaciones: la topológica y la lineal.

Proposición 1.1. Sean $A, B \in \mathbb{C}^{2 \times 2}$, las siguientes afirmaciones son equivalentes:

1. $A \equiv_{\text {ana }} B$.

2. Existe $P \in G L\left(\mathbb{C}^{2}\right)$ tal que $P A=B P$.

3. $A \equiv_{\text {lin }} B$.

La equivalencia 2 . $\Leftrightarrow 3$. de la proposición anterior nos dice que $A \equiv_{\text {lin }} B$ si y sólo si $A$ y $B$ son similares. Ahora bien, del álgebra lineal sabemos que toda matriz $A \in \mathbb{C}^{2 \times 2}$ es similar a su forma canónica de Jordan $J_{A} \in \mathbb{C}^{2 \times 2}$. De esta manera para estudiar el comportamiento cualitativo del flujo asociado a una matriz $A$, es suficiente considerar el flujo asociado a su forma canónica de Jordan $\varphi_{J_{A}}$. El objetivo del presente trabajo es estudiar el comportamiento geométrico de $\varphi_{J_{A}}$. La presentación que haremos esta fuertemente motivada por [7] (ver también [3] y [4]). 


\section{Foliación inducida por un campo lineal}

En el presente trabajo consideraremos matrices inversibles y diagonalizables, luego su forma canónica de Jordan es del tipo $A=\left[\begin{array}{cc}\lambda_{1} & 0 \\ 0 & \lambda_{2}\end{array}\right] \in \mathbb{C}^{2 \times 2}$, donde $\lambda_{1}, \lambda_{2} \in \mathbb{C}^{*}=\mathbb{C}-\{0\}$.

Si $z_{0}=\left(z_{1}^{0}, z_{2}^{0}\right) \in \mathbb{C}^{2}$, sabemos que la única solución $\varphi_{z_{0}}: \mathbb{C} \rightarrow \mathbb{C}^{2}$ del PVI lineal 1 viene dada por $\varphi_{z_{0}}(T)=e^{T A} z_{0}=\left(e^{\lambda_{1} T} z_{1}^{0}, e^{\lambda_{2} T} z_{2}^{0}\right)$. De esta manera, el flujo (global) $\varphi_{A}: \mathbb{C} \times \mathbb{C}^{2} \rightarrow \mathbb{C}^{2}$ asociado al campo lineal $A$, es la función holomorfa

$$
\left.\varphi_{A}(T, z)=e^{T A} z=\left(e^{\lambda_{1} T} z_{1}, e^{\lambda_{2} T} z_{2}\right) \quad \text { (donde } z=\left(z_{1}, z_{2}\right)\right)
$$

Este flujo complejo es una función holomorfa en $\mathbb{C} \times \mathbb{C}^{2}$.

Dado $z=\left(z_{1}, z_{2}\right) \in \mathbb{C}^{2}$ definimos la órbita de $z$ bajo $A$ como

$$
\mathcal{O}_{A}(z)=\left\{\varphi_{A}(T, z) ; T \in \mathbb{C}\right\}
$$

observe que $\mathcal{O}_{A}(z) \subseteq \mathbb{C}^{2}$ es el trazo de la solución de la EDO $z^{\prime}=A z$ que en el instante $T=0$ pasa por $z$. De la unicidad de soluciones de un PVI se desprende que si $z, w \in \mathbb{C}^{2}$ entonces $\mathcal{O}_{A}(z) \cap \mathcal{O}_{A}(w)=\emptyset$ o $\mathcal{O}_{A}(z)=\mathcal{O}_{A}(w)$. Denotemos

$$
\mathcal{F}_{A}=\left\{\mathcal{O}_{A}(z) ; z \in \mathbb{C}^{2}\right\}
$$

observe que los elementos de $\mathcal{F}_{A}$ son curvas complejas disjuntas dos a dos. Más aún, desde que $A \in G L\left(\mathbb{C}^{2}\right)$ entonces el único punto singular del campo $A$ es $z=0$ esto implica que la única órbita que se reduce a un punto es $\mathcal{O}_{A}(0)=\{0\}$. De esta manera la matriz $A$ induce en $\mathbb{C}^{2}-\{0\}$ una familia de curvas complejas unidimensionales, disjuntas dos a dos. $\mathcal{F}_{A}$ es entonces una foliación singular por curvas de $\mathbb{C}^{2}$ inducida por el campo lineal $A$ y sus elementos son también llamados hojas de la foliación.

Es claro que $\{0\}, \mathbb{C}^{*} \times\{0\}$ y $\{0\} \times \mathbb{C}^{*}$ son elementos de $\mathcal{F}_{A}$ (puesto que $\mathcal{O}_{A}(0)=\{0\}$, $\mathcal{O}_{A}(1,0)=\mathbb{C}^{*} \times\{0\}$ y $\left.\mathcal{O}_{A}(0,1)=\{0\} \times \mathbb{C}^{*}\right)$. Surge de manera natural la pregunta: ¿Cómo son los demás elementos de $\mathcal{F}_{A}$ ?

Sea $L=\mathcal{O}_{A}(z) \in \mathcal{F}_{A}-\left\{(0,0), \mathbb{C}^{*} \times\{0\},\{0\} \times \mathbb{C}^{*}\right\}$ esto implica que $z=\left(z_{1}, z_{2}\right) \in \mathbb{C}^{*} \times \mathbb{C}^{*}$. La hoja $L$ está parametrizada por la función analítica (sobreyectiva) $\varphi_{z}: \mathbb{C} \rightarrow L$ dada por

$$
\varphi_{z}(T)=e^{T A} z=\left(e^{\lambda_{1} T} z_{1}, e^{\lambda_{2} T} z_{2}\right)
$$

Si la parametrización $\varphi_{z}$ fuera inyectiva entonces la hoja $L$ sería biholomorfa a $\mathbb{C}$. Vamos entonces a estudiar bajo que condiciones $\varphi_{z}$ es inyectiva.

En primer lugar, observe que

$$
\varphi_{z}\left(T_{1}\right)=\varphi_{z}\left(T_{2}\right) \Longleftrightarrow e^{T_{1} A} z=e^{T_{2} A} z \Longleftrightarrow e^{\left(T_{1}-T_{2}\right) A} z=z \Longleftrightarrow \varphi_{z}\left(T_{1}-T_{2}\right)=z
$$

esto motiva a definir el conjunto

$$
G(z)=\left\{T \in \mathbb{C} ; \varphi_{z}(T)=z\right\}
$$

\section{Observaciones:}

1. $0 \in G(z)$.

2. El cálculo anterior nos dice que

$$
\varphi_{z}\left(T_{1}\right)=\varphi_{z}\left(T_{2}\right) \Longleftrightarrow T_{1}-T_{2} \in G(z)
$$

Concluímos que si $G(z)=\{0\}$ entonces $\varphi_{z}$ es inyectiva. 
3. No es difícil probar que $(G(z),+)$ es un subgrupo abeliano de $(\mathbb{C},+)$.

4. $G(z)$ es llamado grupo estabilizador de $\varphi_{z}$.

De esta manera, nuestro problema se reduce a determinar condiciones bajo las cuales el grupo estabilizador es el trivial. Ahora bien, teniendo en cuenta que $z=\left(z_{1}, z_{2}\right) \in \mathbb{C}^{*} \times \mathbb{C}^{*}$, tenemos

$$
\begin{aligned}
T \in G(z)-\{0\} & \Longleftrightarrow e^{T A} z=z \text { y } T \neq 0 \Longleftrightarrow e^{\lambda_{1} T} z_{1}=z_{1}, e^{\lambda_{2} T} z_{2}=z_{2} \text { y } T \neq 0 \\
& \Longleftrightarrow \text { existen } n_{1}, n_{2} \in \mathbb{Z}^{*} \text { tales que } \lambda_{1} T=2 \pi i n_{1} \text { y } \lambda_{2} T=2 \pi i n_{2} \\
& \Longleftrightarrow \text { existen } n_{1}, n_{2} \in \mathbb{Z}^{*} \text { tales que } \frac{\lambda_{2}}{\lambda_{1}}=\frac{n_{2}}{n_{1}} \Longleftrightarrow \frac{\lambda_{2}}{\lambda_{1}} \in \mathbb{Q}^{*}
\end{aligned}
$$

Resumimos nuestros resultados en el siguiente teorema.

Teorema 2.1. Sea $A=\left[\begin{array}{cc}\lambda_{1} & 0 \\ 0 & \lambda_{2}\end{array}\right] \in \mathbb{C}^{2 \times 2}$, donde $\lambda_{1}, \lambda_{2} \in \mathbb{C}^{*}$ y sea

$$
L \in \mathcal{F}_{A}-\left\{(0,0), \mathbb{C}^{*} \times\{0\},\{0\} \times \mathbb{C}^{*}\right\} .
$$

Las siguientes afirmaciones son equivalentes:

1. L es biholomorfa $a \mathbb{C}$.

2. $\frac{\lambda_{2}}{\lambda_{1}} \notin \mathbb{Q}^{*}$

Observación: Si $\frac{\lambda_{2}}{\lambda_{1}} \in \mathbb{Q}^{*}$, puede demostrarse que $G(z)=2 \pi i \mathbb{Z}$ y que la parametrización $\varphi_{z}: \mathbb{C} \rightarrow L$ induce una inmersión analítica inyectiva del cociente $\mathbb{C} / G(z)$ sobre $L$.

Para estudiar otras propiedades geométricas de las hojas de $\mathcal{F}_{A}$, observamos en primer lugar que ellas son curvas complejas (es decir superficies reales suaves) contenidas en un espacio de dimensión real 4, lo que hace imposible su visualización. Sin embargo, usando convenientemente las propiedades del flujo, podemos entender el su comportamiento geométrico. La idea es la siguiente: sabemos que si $\alpha, \alpha^{\prime} \in \mathbb{C}$ son $\mathbb{R}$-linealmente independientes (no paralelos) entonces cualquier número complejo $T$ puede escribirse como combinación lineal de ellos, es decir, existen $s, t \in \mathbb{R}$ tales que $T=s \alpha+t \alpha^{\prime}$. Ahora bien, las rectas $s \alpha$ y $t \alpha^{\prime}$ son transformadas por el flujo $t \mapsto \varphi_{A}(t, z)$ ( $z$ fijo) en curvas reales contenidas en $\mathbb{R}^{4}$. Conociendo las propiedades geométricas de estas curvas, podemos tener una idea de como se comporta geométricamente la órbita $\mathcal{O}_{A}(z)$, en efecto, basta tener en cuenta que

$$
\varphi_{A}(T, z)=\varphi_{A}\left(s \alpha+t \alpha^{\prime}, z\right)=\varphi_{A}\left(s \alpha, \varphi_{A}\left(t \alpha^{\prime}, z\right)\right)
$$

El razonamiento anterior, motiva el siguiente concepto: dado $\alpha \in \mathbb{C}^{*}$, el flujo $\varphi_{A}$ induce una función $\varphi_{A}^{\alpha}: \mathbb{R} \times \mathbb{C}^{2} \rightarrow \mathbb{C}^{2}$ llamada fujo real inducido por $\varphi_{A}$ en la dirección $\alpha$ definida por

$$
\varphi_{A}^{\alpha}(t, z)=\varphi_{A}(t \alpha, z)=\left(e^{t \alpha \lambda_{1}} z_{1}, e^{t \alpha \lambda_{2}} z_{2}\right)
$$

cuyas órbitas

$$
\mathcal{O}_{\alpha A}(z)=\left\{\varphi_{A}^{\alpha}(t, z) ; t \in \mathbb{R}\right\}
$$

son curvas reales contenidas en $\mathcal{O}_{A}(z)$.

Fijemos un $z=\left(z_{1}, z_{2}\right) \in \mathbb{C}^{*} \times \mathbb{C}^{*}$, observe que si denotamos $\alpha=\hat{\lambda}_{1}=\frac{2 \pi i}{\lambda_{1}}$ entonces

$$
\varphi_{A}^{\hat{\lambda}_{1}}(t, z)=\left(e^{2 \pi i t} z_{1}, e^{2 \pi i t \lambda_{2} / \lambda_{1}} z_{2}\right)
$$


Se sigue que el cilindro $\Gamma_{1, r}=\partial D_{r}[0] \times \mathbb{C}$ (donde $\left|z_{1}\right|=r>0$ ) es invariante por $\varphi_{A}^{\hat{\lambda}_{1}}$ (es decir $\left.\varphi_{A}^{\hat{\lambda}_{1}}(t, z) \in \Gamma_{1, r}, \forall t \in \mathbb{R}\right)$. Geométricamente esto significa que la curva $t \mapsto \varphi_{A}^{\hat{\lambda}_{1}}(t, z)$ está contenida en el cilindro $\Gamma_{1, r}$. Más aún, no es difícil probar que

$$
\mathcal{O}_{A}(z) \cap \Gamma_{1, r}=\mathcal{O}_{\hat{\lambda}_{1} A}(z)
$$

De esta manera, describir las órbitas de $\varphi_{A}^{\hat{\lambda}_{1}}$ es describir las intersecciones de los elementos de $\mathcal{F}_{A}$ con $\Gamma_{1, r}$.

Denotemos por $\mu=\left(\mu_{1}, \mu_{2}\right): \mathbb{R} \rightarrow \Gamma_{1, r}$ a la parametrización de $\mathcal{O}_{\hat{\lambda}_{1} A}(z)$, es decir

$$
\mu(t)=\varphi_{A}^{\hat{\lambda}_{1}}(t, z)=\left(e^{2 \pi i t} z_{1}, e^{2 \pi i t \lambda_{2} / \lambda_{1}} z_{2}\right)=\left(\mu_{1}(t), \mu_{2}(t)\right)
$$

Se presentan tres casos mutuamente excluyentes:

Caso 1: $\frac{\lambda_{2}}{\lambda_{1}} \notin \mathbb{R}$. Esto implica que $\operatorname{Im}\left(\frac{\lambda_{2}}{\lambda_{1}}\right) \neq 0$, luego

$$
\left|\mu_{2}(t)\right|=\left|e^{2 \pi i t \lambda_{2} / \lambda_{1}} z_{2}\right|=e^{-2 \pi \operatorname{Im}\left(\lambda_{2} / \lambda_{1}\right) t} \cdot\left|z_{2}\right|, \cdot \quad \forall t \in \mathbb{R}
$$

se sigue que

$$
\lim _{t \rightarrow+\infty}\left|\mu_{2}(t)\right|=\left\{\begin{array}{ll}
0, & \text { si } \operatorname{Im}\left(\lambda_{2} / \lambda_{1}\right)>0 \\
+\infty, & \text { si } \operatorname{Im}\left(\lambda_{2} / \lambda_{1}\right)<0
\end{array} \quad \text { y } \lim _{t \rightarrow-\infty}\left|\mu_{2}(t)\right|= \begin{cases}+\infty, & \text { si } \operatorname{Im}\left(\lambda_{2} / \lambda_{1}\right)>0 \\
0, & \text { si } \operatorname{Im}\left(\lambda_{2} / \lambda_{1}\right)<0\end{cases}\right.
$$

Concluimos que $\mu$ es una curva abierta, que se enrrolla alrededor del cilindro $\Gamma_{1, r}$ alejándose o acercándose al plano $\mathbb{C} \times\{0\}$ según el signo de $\operatorname{Im}\left(\frac{\lambda_{2}}{\lambda_{1}}\right)$.

Caso 2: $\frac{\lambda_{2}}{\lambda_{1}} \in \mathbb{Q}$. Denotemos $\frac{\lambda_{2}}{\lambda_{1}}=\frac{m}{n}$ donde $m$ y $n$ son enteros coprimos (donde $n>0$ ). Observe que

$$
\mu(t)=\left(e^{2 \pi i t} z_{1}, e^{2 \pi i t m / n} z_{2}\right) \in \partial D_{\left|z_{1}\right|}[0] \times \partial D_{\left|z_{2}\right|}[0] \subseteq \Gamma_{1, r}
$$

y

$$
\mu(n)=\left(e^{2 n \pi i} z_{1}, e^{2 m \pi i} z_{2}\right)=\left(z_{1}, z_{2}\right)
$$

es decir que después de $n$ unidades de tiempo, la curva llega al punto inicial. Concluimos que $\mu$ es una curva cerrada contenida en el toro $D_{\left|z_{1}\right|}[0] \times \partial D_{\left|z_{2}\right|}[0]$.

Caso 3: $\frac{\lambda_{2}}{\lambda_{1}} \in \mathbb{R}-\mathbb{Q}$. Se cumple

$$
\left|\mu_{2}(t)\right|=\left|e^{2 \pi i t \lambda_{2} / \lambda_{1}} z_{2}\right|=\left|z_{2}\right|, \quad \forall t \in \mathbb{R}
$$

Concluimos que $\mu$ es una curva en el toro $D_{\left|z_{1}\right|}[0] \times \partial D_{\left|z_{2}\right|}[0]$ que no se cierra nunca pero que está arbitrariamente cerca de cualquier punto del toro.

Resumimos nuestros resultados en el siguiente teorema.

Teorema 2.2. Sea $A=\left[\begin{array}{cc}\lambda_{1} & 0 \\ 0 & \lambda_{2}\end{array}\right] \in \mathbb{C}^{2 \times 2}$, con $\lambda_{1}, \lambda_{2} \in \mathbb{C}^{*}$ y denotemos $\hat{\lambda}_{1}=\frac{2 \pi i}{\lambda_{1}}$. Sea $L \in \mathcal{F}_{A}-\left\{(0,0), \mathbb{C}^{*} \times\{0\},\{0\} \times \mathbb{C}^{*}\right\}$ y $z=\left(z_{1}, z_{2}\right) \in \mathbb{C}^{*} \times \mathbb{C}^{*}$ tal que L $=\mathcal{O}(z)$. Denotemos por $\mu: \mathbb{R} \rightarrow \mathbb{C}^{2}$ al flujo real inducido $\mu(t)=\varphi_{A}^{\hat{\lambda}_{1}}(t, z)$ el cual parametriza la hoja $\mathcal{O}_{\hat{\lambda}_{1} A}(z) \subseteq L$. 
1. Si $\frac{\lambda_{2}}{\lambda_{1}} \notin \mathbb{R}$ entonces $\mu$ es una curva abierta, que se enrrolla alrededor del cilindro $\Gamma_{1}=$ $\partial D_{\left|z_{1}\right|}[0] \times \mathbb{C}$ alejándose o acercándose al plano $\mathbb{C} \times\{0\}$ según el signo de $\operatorname{Im}\left(\frac{\lambda_{2}}{\lambda_{1}}\right)$.

2. Si $\frac{\lambda_{2}}{\lambda_{1}} \in \mathbb{Q}$ entonces $\mu$ es una curva cerrada contenida en el toro $\partial D_{\left|z_{1}\right|}[0] \times \partial D_{\left|z_{2}\right|}[0] \subseteq \Gamma_{1}$.

3. Si $\frac{\lambda_{2}}{\lambda_{1}} \in \mathbb{R}-\mathbb{Q}$ entonces $\mu$ es una curva abierta densa en el toro $\partial D_{\left|z_{1}\right|}[0] \times \partial D_{\left|z_{2}\right|}[0] \subseteq \Gamma_{1}$.

Como consecuencia del teorema anterior, tenemos las siguientes propiedades topológicas de las hojas de $\mathcal{F}_{A}$.

Corolario Sea $L \in \mathcal{F}_{A}-\left\{(0,0), \mathbb{C}^{*} \times\{0\},\{0\} \times \mathbb{C}^{*}\right\}$ y sea $z=\left(z_{1}, z_{2}\right) \in \mathbb{C}^{*} \times \mathbb{C}^{*}$ tal que $L=\mathcal{O}(z)$.

1. Si $\frac{\lambda_{2}}{\lambda_{1}} \notin \mathbb{R}$ entonces $\mathbb{C}^{*} \times\{0\} \subseteq \bar{L}$.

2. Si $\frac{\lambda_{2}}{\lambda_{1}} \in \mathbb{R}-\mathbb{Q}$ entonces $D_{\left|z_{1}\right|}[0] \times \partial D_{\left|z_{2}\right|}[0] \subseteq \bar{L}$.

De esta manera, hemos determinado el comportamiento geométrico de la curva real contenida en la órbita, determinada por la dirección $\hat{\lambda}_{1}$. Para lograr nuestro objetivo, necesitamos determinar una segunda dirección no paralela a $\hat{\lambda}_{1}$. Como veremos en la siguiente sección, la elección de esta segunda dirección, depende de la posición que ocupan los autovalores de $\lambda_{1}$ y $\lambda_{2}$ de $A$.

\section{Dominio de Poincaré y Dominio de Siegel}

Definición 3.1. Decimos que el par $\left(\lambda_{1}, \lambda_{2}\right) \in \mathbb{C}^{*} \times \mathbb{C}^{*}$. está en el dominio de Poincaré si y sólo si $\frac{\lambda_{2}}{\lambda_{1}} \notin \mathbb{R}^{-}$, caso contrario diremos que $\left(\lambda_{1}, \lambda_{2}\right)$ está en el dominio de Siegel.

Usaremos la siguiente notación:

$$
\begin{aligned}
& \mathcal{D}_{P}=\left\{\left(\lambda_{1}, \lambda_{2}\right) \in \mathbb{C}^{*} \times \mathbb{C}^{*} ; \frac{\lambda_{2}}{\lambda_{1}} \notin \mathbb{R}^{-}\right\} \\
& \mathcal{D}_{S}=\left\{\left(\lambda_{1}, \lambda_{2}\right) \in \mathbb{C}^{*} \times \mathbb{C}^{*} ; \frac{\lambda_{2}}{\lambda_{1}} \in \mathbb{R}^{-}\right\}
\end{aligned}
$$

Observe que $\mathbb{C}^{*} \times \mathbb{C}^{*}$ es unión disjunta de $\mathcal{D}_{P}$ y $\mathcal{D}_{S}$.

Decimos que $A \in \mathbb{C}^{2 \times 2}$ está en el dominio de Poincaré (resp. Siegel) si y sólo si sus autovalores están en el dominio de Poincaré (resp. Siegel).

Los dos resultados siguientes establecen definiciones equivalentes del dominio de Poincaré y del dominio de Siegel las cuales tienen un significado geométrico muy sencillo y sus demostraciones puedn ser encontradas en [3]:

Proposición 3.1. Sea $\left(\lambda_{1}, \lambda_{2}\right) \in \mathbb{C}^{*} \times \mathbb{C}^{*}$. Las siguientes afirmaciones son equivalentes:

1. $\left(\lambda_{1}, \lambda_{2}\right) \in \mathcal{D}_{S}$.

2. $0 \in] \lambda_{1}, \lambda_{2}[$. 
3. $\left|\operatorname{Arg}\left(\lambda_{1}\right)-\operatorname{Arg}\left(\lambda_{2}\right)\right|=\pi$.

Corolario. Sea $\left(\lambda_{1}, \lambda_{2}\right) \in \mathbb{C}^{*} \times \mathbb{C}^{*}$. Las siguientes afirmaciones son equivalentes:

1. $\left(\lambda_{1}, \lambda_{2}\right) \in \mathcal{D}_{P}$.

2. $0 \notin] \lambda_{1}, \lambda_{2}[$.

3. $\left|\operatorname{Arg}\left(\lambda_{1}\right)-\operatorname{Arg}\left(\lambda_{2}\right)\right| \neq \pi$.

Las dos proposiciones siguientes, cuyas demostraciones son inmediatas, serán útiles posteriormente.

Proposición 3.2. Si $\left(\lambda_{1}, \lambda_{2}\right) \in \mathcal{D}_{P}$ entonces existe $\alpha \in S^{1}$ tales que $\operatorname{Re}\left(\alpha \lambda_{1}\right)<0$ y $\operatorname{Re}\left(\alpha \lambda_{2}\right)<0$.

Observación: Geométricamente la proposición anterior nos dice que si $\lambda_{1}$ y $\lambda_{2}$ están en el dominio de Poincaré, entonces por una rotación adecuada podemos enviarlos al segundo o tercer cuadrante.

Proposición 3.3. Si $\left(\lambda_{1}, \lambda_{2}\right) \in \mathcal{D}_{S}$ entonces existe $\alpha \in S^{1}$ tales que $\alpha \lambda_{1}, \alpha \lambda_{2} \in \mathbb{R} y\left(\alpha \lambda_{1}\right)\left(\alpha \lambda_{2}\right)<$ 0 .

El siguiente resultado establece que tan abundante es $D_{S}$ en $\mathbb{C}^{*} \times \mathbb{C}^{*}$.

Teorema 3.1. $D_{S}$ es una superficie suave de dimensión real 3 que cumple la siguiente propiedad

$$
\left(\lambda_{1}, \lambda_{2}\right) \in D_{S}, t \in \mathbb{R} \Longrightarrow t\left(\lambda_{1}, \lambda_{2}\right) \in D_{S}
$$

(i.e. $D_{S}$ es un cono con vértice el origen de $\mathbb{C}^{2}$ ).

Demostración. En primer lugar, observe que (3) es evidente. Por otro lado, se cumple

$$
\frac{\lambda_{2}}{\lambda_{1}}=\frac{\lambda_{2} \bar{\lambda}_{1}}{\left|\lambda_{1}\right|^{2}}
$$

Esto motiva a definir los conjuntos

$$
\stackrel{A}{=}=\left\{\left(\lambda_{1}, \lambda_{2}\right) \in \mathbb{C}^{*} \times \mathbb{C}^{*} ; \operatorname{Im}\left(\lambda_{2} \bar{\lambda}_{1}\right)=0\right\} \quad \text { y } \quad B=\left\{\left(\lambda_{1}, \lambda_{2}\right) \in \mathbb{C}^{*} \times \mathbb{C}^{*} ; \operatorname{Re}\left(\lambda_{2} \bar{\lambda}_{1}\right)<0\right\}
$$

Se sigue inmediatamente que $D_{S}=A \cap B$.

Si definimos $\phi: \mathbb{C}^{*} \times \mathbb{C}^{*} \rightarrow \mathbb{R}$ como $\phi\left(\lambda_{1}, \lambda_{2}\right)=\operatorname{Re}\left(\lambda_{2} \bar{\lambda}_{1}\right)$, se sigue que $\phi$ es continua y por tanto $B=\phi^{-1}(]-\infty, 0[)$ es abierto en $\mathbb{C}^{*} \times \mathbb{C}^{*}$. De esta manera tenemos que $D_{S}$ es abierto en $A$. Afirmación: $A \subseteq \mathbb{C}^{*} \times \mathbb{C}^{*}$ es una superficie suave de dimensión real 3. En efecto, denotemos $U=\mathbb{C}^{*} \times \mathbb{C}^{*}, \lambda_{1}=x_{1}+i x_{2}$ y $\lambda_{2}=x_{3}+i x_{4}$. Podemos observar $U$ como un abierto de $\mathbb{R}^{4}$ y podemos identificar el par $\left(\lambda_{1}, \lambda_{2}\right) \in \mathbb{C}^{2}$ con $\left(x_{1}, x_{2}, x_{3}, x_{4}\right) \in \mathbb{R}^{4}$. Se sigue entonces que $\operatorname{Im}\left(\lambda_{2} \bar{\lambda}_{1}\right)=x_{1} x_{4}-x_{2} x_{3}$. Esto motiva a considerar la función suave $f: U \rightarrow \mathbb{R}$ definida por $f\left(x_{1}, x_{2}, x_{3}, x_{4}\right)=x_{1} x_{4}-x_{2} x_{3}$. . .

Observe que $\nabla f\left(x_{1}, x_{2}, x_{3}, x_{4}\right)=\left(x_{4},-x_{3},-x_{2}, x_{1}\right) \neq(0,0,0,0), \forall\left(x_{1}, x_{2}, x_{3}, x_{4}\right) \in U$. Concluímos que 0 es un valor regular de $f$ y por tanto $A=f^{-1}(0) \subseteq U$ es una superficie suave de dimensión real 3. Esto prueba la Afirmación.

Finalmente como $D_{S}$ es abierto en $A$ entonces $D_{S}$ también es una superficie suave de dimensión real 3.

Como consecuencia inmediata del Teorema 3.1 tenemos los siguientes resultados.

Corolario 1. $D_{S}$ tiene interior vacío.

Corolario 2. $D_{P}$ es abierto y denso en $\mathbb{C}^{*} \times \mathbb{C}^{*}$. 


\section{Estudio geométrico de las órbitas en el dominio de Poincaré}

Retomando el problema de la Sección 2, si $A=\left[\begin{array}{cc}\lambda_{1} & 0 \\ 0 & \lambda_{2}\end{array}\right] \in \mathbb{C}^{2 \times 2} \operatorname{con}\left(\lambda_{1}, \lambda_{2}\right) \in \mathcal{D}_{P}$, vamos a probar que podemos elegir una segunda dirección $\mathbb{R}$-linealmente independiente con $\hat{\lambda}_{1}$ tal que sea fácil determinar el comportamiento geométrico de la curva imagen bajo el flujo de la recta real generada por esta dirección.

En efecto, por la Proposición 3.2, existe $\alpha \in S^{1}$ tales que $\operatorname{Re}\left(\alpha \lambda_{1}\right)<0$ y $\operatorname{Re}\left(\alpha \lambda_{2}\right)<0$. Denotando $\alpha \lambda_{1}=a+i b$ y $\alpha \lambda_{2}=c+i d$, la matriz compleja $\alpha A=\left[\begin{array}{cc}\alpha \lambda_{1} & 0 \\ 0 & \alpha \lambda_{2}\end{array}\right] \in \mathbb{C}^{2 \times 2}$ puede ser identificada con la matriz real (a la que también llamaremos $\alpha A$ )

$$
\left[\begin{array}{cccc}
a & -b & 0 & 0 \\
b & a & 0 & 0 \\
0 & 0 & c & -d \\
0 & 0 & d & c
\end{array}\right] \in \mathbb{R}^{4 \times 4}
$$

la cual tiene todos sus autovalores con parte real negativa. Se sigue que el origen de $\mathbb{C}^{2}=\mathbb{R}^{4}$ es un atractor para la matriz real $\alpha A$ (ver [2], [10]), es decir

$$
\lim _{t \rightarrow \infty} \varphi_{\alpha A}(t, z)=0, \quad \forall z \in \mathbb{C}^{2}
$$

En particular, se șigue que el origen de $\mathbb{C}^{2}$ está en la adherencia de $\mathcal{O}_{A}(z), \forall z \in \mathbb{C}^{2}$. Más aún, un fácil cálculo muestra que el flujo (real) asociado $\varphi_{\alpha A}: \mathbb{R} \times \mathbb{R}^{4} \rightarrow \mathbb{R}^{4}$ a la matriz $\alpha A$ en cualquier punto $z \in \mathbb{C}^{2}=\mathbb{R}^{4}$ viene dado por

$$
\varphi_{\alpha A}(t, z)=e^{t(\alpha A)} z=\left(e^{\lambda_{1} \alpha t} z_{1}, e^{\lambda_{2} \alpha t} z_{2}\right)=\varphi_{A}^{\alpha}(t, z)
$$

es decir $\varphi_{\alpha A}$ puede ser identificado con el flujo real $\varphi_{A}^{\alpha}: \mathbb{R} \times \mathbb{C}^{2} \rightarrow \mathbb{C}^{2}$ inducido por $\varphi_{A}$ en la dirección $\alpha$. Además, es claro que $\alpha$ no es paralelo a $\hat{\lambda}_{1}$.

Para demostrar otra interesante propiedad geométrica de las hojas en el dominio de Poincaré, necesitamos definir un caso particular del concepto de transversalidad.

Dado $r>0$, denotemos por $S_{r}^{3}$ a la esfera

$$
S_{r}^{3}=\left\{z=\left(z_{1}, z_{2}\right) \in \mathbb{C}^{2} ;\left|z_{1}\right|^{2}+\left|z_{2}\right|^{2}=r^{2}\right\}
$$

Podemos observar $S_{r}^{3}$ como una superficie suave (de clase $C^{\infty}$ ) de $\mathbb{R}^{4}$, luego el espacio tangente a $S_{r}^{3}$ en $z \in S_{r}^{3}$, denotado por $T_{z}\left(S_{r}^{3}\right)$ viene dado por

$$
T_{z}\left(S_{r}^{3}\right)=\left\{w \in \mathbb{R}^{4} ;\langle w, z\rangle_{\mathbb{R}}=0\right\}=\{z\}^{\perp}
$$

en donde $\langle,\rangle_{\mathbb{R}}$ denota el producto interno real de $z$ y $w$ considerados como puntos de $\mathbb{R}^{4}$. Ahora bien, sabemos que $\mathbb{C}^{2}$ tiene su producto interno $\langle,\rangle_{\mathbb{C}}$ definido por

$$
\langle z, w\rangle_{\mathbb{C}}=\left\langle\left(z_{1}, z_{2}\right),\left(w_{1}, w_{2}\right)\right\rangle_{\mathbb{C}}=z_{1} \bar{w}_{1}+z_{2} \bar{w}_{2}
$$

Un fácil cálculo muestra que

$$
\langle z, w\rangle_{\mathbb{R}}=\operatorname{Re}\left(\langle z, w\rangle_{\mathbb{C}}\right)
$$

luego

$$
T_{z}\left(S_{r}^{3}\right)=\left\{w \in \mathbb{C}^{2} ; \operatorname{Re}\left(\langle w, z\rangle_{\mathbb{C}}\right)=0\right\}
$$


Por otro lado, sea $\varphi: D \rightarrow \mathbb{C}^{2}$ una función holomorfa definida en el disco abierto $D \subseteq \mathbb{C}^{2}$ tal que $\varphi^{\prime}(T) \neq 0, \forall T \in D$. En estas condiciones su trazo $L=\{\varphi(T) ; T \in D\}$ es una curva compleja holomorfa en $\mathbb{C}^{2}$. Si $T \in D$ es tal que $\varphi(T)=z$ entonces el espacio tangente a $L$ en el punto $z$ es el espacio vectorial complejo $T_{z} L$ generado por $\varphi^{\prime}(T)$, es decir

$$
T_{z} L=\left\{a \varphi^{\prime}(T) ; a \in \mathbb{C}\right\}
$$

La curva compleja $L$ puede ser observada como una superficie real bidimensional de clase $C^{\infty}$ contenida en $\mathbb{R}^{4}$ y su espacio tangente $T_{z} L$ sería un subespacio vectorial de $\mathbb{R}^{4}$ de dimensión (real) dos.

Decimos que la curva holomorfa $L$ es transversal a la esfera $S_{r}^{3}$ si y sólo si

$$
T_{z}\left(S_{r}^{3}\right)+T_{z} L=\mathbb{R}^{4} \quad \forall z \in S_{r}^{3} \cap L .
$$

Vamos a probar que cualquier hoja de $\mathcal{F}_{A}$ intersecta transversalmente a la esfera $S_{r}^{3}$. En efecto, sea $L \in \mathcal{F}_{A}-\{0\}$ entonces existe $z_{0} \in \mathbb{C}^{2}-\{0\}$ tal que $L=\mathcal{O}_{A}\left(z_{0}\right)$. Tomemos cualquier $z \in L \cap S_{r}^{3}$, luego existe $T_{0} \in \mathbb{C}$ tal que $\varphi_{z_{0}}\left(T_{0}\right)=z$. Por otro lado, sabemos que $L$ es una curva compleja holomorfa parametrizada por $\varphi_{z_{0}}$, luego su espacio tangente en $z=\varphi_{z_{0}}\left(T_{0}\right)$ viene dado por

$$
T_{z} L=\left\{a \varphi_{z_{0}}^{\prime}\left(T_{0}\right) ; a \in \mathbb{C}\right\}=\{a A z ; a \in \mathbb{C}\}
$$

Tomando $a=\alpha \in S^{1}$ (donde $\operatorname{Re}\left(\alpha \lambda_{1}\right)<0$ y $\operatorname{Re}\left(\alpha \lambda_{2}\right)<0$ y $c \in \mathbb{R}$ tal que máx $\left\{\operatorname{Re}\left(\alpha \lambda_{1}\right), \operatorname{Re}\left(\alpha \lambda_{2}\right)\right\}<c<0$, tenemos

$$
\begin{aligned}
\operatorname{Re}\left(\langle\alpha A z, z\rangle_{\mathbb{C}}\right) & =\operatorname{Re}\left(\left\langle\left(\alpha \lambda_{1} z_{1}, \alpha \lambda_{2} z_{2}\right),\left(z_{1}, z_{2}\right)\right\rangle_{\mathbb{C}}\right)=\operatorname{Re}\left(\alpha \lambda_{1}\left|z_{1}\right|^{2}+\alpha \lambda_{2}\left|z_{2}\right|^{2}\right) \\
& =\operatorname{Re}\left(\alpha \lambda_{1}\right)\left|z_{1}\right|^{2}+\operatorname{Re}\left(\alpha \lambda_{2}\right)\left|z_{2}\right|^{2}<c r^{2}<0
\end{aligned}
$$

Se desprende que $\alpha A z \notin T_{z}\left(S_{r}^{3}\right)$ y por tanto

$$
T_{z}\left(S_{r}^{3}\right)+T_{z} L=\mathbb{R}^{4}, \quad \forall z \in S_{r}^{3} \cap L .
$$

Resumimos nuestros resultados en el siguiente teorema.

Teorema 4.1. Si $A=\left[\begin{array}{cc}\lambda_{1} & 0 \\ 0 & \lambda_{2}\end{array}\right] \in \mathbb{C}^{2 \times 2} \operatorname{con}\left(\lambda_{1}, \lambda_{2}\right) \in \mathcal{D}_{P}$ entonces

1. Existe un flujo real (lineal) cuyas hojas están contenidas en las hojas de $\mathcal{F}_{A}$ y para el cual $(0,0) \in \mathbb{C}^{2}$ es un atractor.

2. $(0,0) \in \bar{L}, \forall L \in \mathcal{F}_{A}$.

3. Las hojas de $\mathcal{F}_{A}-\{(0,0)\}$ son transversales a la familia de esferas $\left\{S_{r}^{3}\right\}_{r>0}$.

\section{Observaciones:}

1. De la topologia diferencial (ver [8], [9]) se sabe que si $M, N \subseteq \mathbb{R}^{n}$ son superficies de clase $C^{k}$ que se intersectan transversalmente entonces $M \cap N$ es una superficie de clase $C^{k}$ cuya codimensión es dada por

$$
\operatorname{codim}(M \cap N)=\operatorname{codim} M+\operatorname{codim} N
$$

donde $\operatorname{codim} M=n-\operatorname{dim} M$.

En nuestro caso, como $L, S_{r}^{3} \subseteq \mathbb{R}^{4}$ son superficies de clase $C^{\infty}$ de dimensiones 2 y 3 , respectivamente entonces $L \cap S_{r}^{3} \subseteq \mathbb{R}^{4}$ es una superficie de clase $C^{\infty}$ de codimensión

$$
\operatorname{codim}\left(L \cap S_{r}^{3}\right)=\operatorname{codim}(L)+\operatorname{codim}\left(S_{r}^{3}\right)=2+1=3
$$

es decir $L \cap S_{r}^{3}$ es una curva de clase $C^{\infty}$ contenida en la esfera $S_{r}^{3}$. 
2. Por la observación anterior, la foliación compleja $\mathcal{F}_{A}$ induce una foliación por curvas de clase $C^{\infty}$ en la esfera $S_{r}^{3}$ obtenida al interceptar las hojas de $\mathcal{F}_{A}$ con $S_{r}^{3}$.

3. Decimos que $L \in \mathcal{F}_{A}$ es una hoja de Poincaré (o separatriz) si y sólo si $(0,0) \in \bar{L}$. El teorema anterior muestra que si $A=\operatorname{diag}\left[\lambda_{1}, \lambda_{2}\right] \in \mathbb{C}^{2 \times 2}$. con $\left(\lambda_{1}, \lambda_{2}\right) \in \mathcal{D}_{P}$ entonces toda $L \in \mathcal{F}_{A}$ es una hoja de Poincaré

Sabemos que si $L \in \mathcal{F}_{A}-\{(0,0)\}$ entonces $L \cap S^{3}$ es una curva de clase $C^{\infty}$ contenida en la esfera unitaria $S_{1}^{3}=S^{3}$ ¿Podemos conocer algunas propiedades geométricas de esta curva?

Sea $\alpha \in S^{1}$ tal que $\operatorname{Re}\left(\alpha \lambda_{1}\right)<0$ y $\operatorname{Re}\left(\alpha \lambda_{2}\right)<0$, dado $z=\left(z_{1}, z_{2}\right) \in \mathbb{C}^{2}-\{(0,0)\}=U$, sabemos que $\mathcal{O}_{\alpha A}(z)=\left\{\varphi_{A}(t \alpha, z) ; t \in \mathbb{R}\right\}$ corta transversalmente a $S^{3}$ en un único punto, es decir, existe un único $s=s(z) \in \mathbb{R}$ tal que $\varphi_{A}(s(z) \alpha, z) \in S^{3}$.

De esta manera, hemos construído la función $s: U \rightarrow \mathbb{R}$ definida implícitamente por la relación

$$
\left\|\varphi_{A}(s(z) \alpha, z)\right\|^{2}=1
$$

Como $\varphi_{A}(s(z) \alpha, z)=\left(e^{s(z) \alpha \lambda_{1}} z_{1}, e^{s(z) \alpha \lambda_{2}} z_{2}\right)$, de (4) se sigue que

$$
e^{2 s(z) \operatorname{Re}\left(\alpha \lambda_{1}\right)}\left|z_{1}\right|^{2}+e^{2 s(z) \operatorname{Re}\left(\alpha \lambda_{2}\right)}\left|z_{2}\right|^{2}=1
$$

Identificando $U$ como un abierto de $\mathbb{R}^{4}, z_{1}=\left(x_{1}, x_{2}\right) \in \mathbb{R}^{2}, z_{2}=\left(x_{3}, x_{4}\right) \in \mathbb{R}^{2}$ y denotando $a_{1}=\operatorname{Re}\left(\alpha \lambda_{1}\right), a_{2}=\operatorname{Re}\left(\alpha \lambda_{2}\right)$, motivados por (5), consideramos la función $f: U \times \mathbb{R} \rightarrow \mathbb{R}$ definida por

$$
f(z, s)=e^{2 a_{1} s}\left(x_{1}^{2}+x_{2}^{2}\right)+e^{2 a_{2} s}\left(x_{3}^{2}+x_{4}^{2}\right)
$$

Claramente $f$ es de clase $C^{\infty}$ en su dominio. Sea $\left(z_{0}, s_{0}\right) \in U \times \mathbb{R}$ tal que $f\left(z_{0}, s_{0}\right)=1$, se tiene que

$$
\frac{\partial f}{\partial s}\left(z_{0}, s_{0}\right)=2 a_{1} e^{2 a_{1} s}\left(x_{1}^{2}+x_{2}^{2}\right)+2 a_{2} e^{2 a_{2} s}\left(x_{3}^{2}+x_{4}^{2}\right)<0
$$

Por el Teorema de la función impícita, existen vecindades abierta $B \subseteq U$ de $z_{0}, I \subseteq \mathbb{R}$ de $s_{0}$ y existe una función de clase $C^{\infty} s: B \rightarrow I$ tal que $f(z, s(z))=1, \forall z \in B$.

Concluímos que la función $s: U \rightarrow \mathbb{R}$ es de clase $C^{\infty}$ en su dominio.

A continuación, consideremos el cilindro $\Gamma_{1}=S^{1} \times \mathbb{C}$ el cual puede ser observado como una superficie real $S^{1} \times \mathbb{R}^{2}$ de dimensión 3 y clase $C^{\infty}$. Su espacio tangente en cualquier punto $z=\left(z_{1}, z_{2}\right) \in \Gamma_{1}$ viene dado por

$$
\begin{aligned}
T_{z}\left(\Gamma_{1}\right) & =T_{z}\left(S^{1} \times \mathbb{R}^{2}\right)=T_{z_{1}}\left(S^{1}\right) \times T_{z_{2}} \mathbb{R}^{2}=\left\{w_{1} \in \mathbb{C} ;\left\langle w_{1}, z_{1}\right\rangle_{\mathbb{R}}=0\right\} \times \mathbb{R}^{2} \\
& =\left\{w_{1} \in \mathbb{C} ; \operatorname{Re}\left(w_{1} \bar{z}_{1}\right)=0\right\} \times \mathbb{R}^{2} \\
& =\left\{w=\left(w_{1}, w_{2}\right) \in \mathbb{C}^{2} ; \operatorname{Re}\left(w_{1} \bar{z}_{1}\right)=0\right\}
\end{aligned}
$$

Decimos que la curva holomorfa $L$ es transversal al cilindro $\Gamma_{1}$ si y sólo si

$$
T_{z}\left(\Gamma_{1}\right)+T_{z} L=\mathbb{R}^{4}, \quad \forall z \in \Gamma_{1} \cap L .
$$

No es difícil probar el siguiente resultado.

Lema 4.1. Sea $A=\left[\begin{array}{cc}\lambda_{1} & 0 \\ 0 & \lambda_{2}\end{array}\right] \in \mathbb{C}^{2 \times 2} \operatorname{con}\left(\lambda_{1}, \lambda_{2}\right) \in \mathcal{D}_{P}$. Si $L \in \mathcal{F}_{A}-\left\{\{(0,0)\},\{0\} \times \mathbb{C}^{*}\right\}$ entonces $L$ es transversal a $\Gamma_{1}$.

Por el lema anterior $L \cap \Gamma_{1}$ es una curva (real) de clase $C^{\infty}$ contenida en el cilindro $\Gamma_{1}$, el siguiente resultado muestra que es fácil parametrizar esta curva 
Lema 4.2. Sea $A=\operatorname{diag}\left[\lambda_{1}, \lambda_{2}\right] \in \mathbb{C}^{2 \times 2} \operatorname{con}\left(\lambda_{1}, \lambda_{2}\right) \in \mathcal{D}_{P}$. Si $L \in \mathcal{F}_{A}-\left\{\{(0,0)\},\{0\} \times \mathbb{C}^{*}\right\}$ entonces

$$
L \cap \Gamma_{1}=\left\{\varphi_{A}^{\widehat{\lambda}_{1}}(t, z) ; t \in \mathbb{R}\right\}
$$

donde $z$ es cualquier punto de $L \cap \Gamma_{1}$.

Demostración. Tomemos $z=\left(z_{1}, z_{2}\right) \in L \cap \Gamma_{1}$, luego $\left|z_{1}\right|=1$. Dado $t \in \mathbb{R}$ se tiene que $\varphi_{A}^{\hat{\lambda}_{1}}(t, z)=\left(e^{2 \pi i t} z_{1}, e^{2 \pi i t \lambda_{2} / \lambda_{1}} z_{2}\right) \in \mathcal{O}_{A}(z)=L$ y como

$$
\left|e^{2 \pi i t} z_{1}\right|=\left|z_{1}\right|=1, \quad \forall t \in \mathbb{R},
$$

concluimos que $\left\{\varphi_{A}^{\hat{\lambda}_{1}}(t, z) ; t \in \mathbb{R}\right\} \subseteq L \cap \Gamma_{1}$. Recíprocamente, sea $w=\left(w_{1}, w_{2}\right) \in \mathcal{O}_{A}(z) \cap \Gamma_{1}$ entonces existe $T_{0} \in \mathbb{C}$ tal que $w=\varphi_{A}\left(T_{0}, z\right)=\left(e^{T_{0} \lambda_{1}} z_{1}, e^{T_{0} \lambda_{2}} z_{2}\right)$ y como $w \in \Gamma_{1}$, se tiene que

$$
1=\left|w_{1}\right|=\left|e^{T_{0} \lambda_{1}} z_{1}\right|=\left|e^{T_{0} \lambda_{1}}\right| \cdot\left|z_{1}\right|=e^{\operatorname{Re}\left(T_{0} \lambda_{1}\right)}
$$

Se sigue que $\operatorname{Re}\left(T_{0} \lambda_{1}\right)=0$, es decir $T_{0}=c \frac{i}{\lambda_{1}}=t \widehat{\lambda}_{1}$. Por tanto

$$
w=\varphi_{A}\left(t \widehat{\lambda}_{1}, z\right)=\varphi_{A}^{\widehat{\lambda_{1}}}(t, z)
$$

Esto prueba que $L \cap \Gamma_{1} \subseteq\left\{\varphi_{A}^{\hat{\lambda}_{1}}(t, z) ; t \in \mathbb{R}\right\}$.

Acabamos de parametrizar la curva que es intersección de una hoja de la foliación con el cilindro $\Gamma_{1}$. Por lo realizado en la sección anterior, conocemos las propiedades de esta curva, vamos a ver como esto nos ayuda a comprender la geometría de la curva obtenida al intersectar la misma hoja con la esfera unitaria $S^{3}$.

Denotemos por $X=S^{3}-\left\{\left(z_{1}, z_{2}\right) \in \mathbb{C}^{2} ; z_{1}=0\right\}=S^{3}-\left(\{0\} \times \mathbb{C}^{*}\right)$ y definamos una función $H: \Gamma_{1} \rightarrow X$ del modo siguiente: Sea $\alpha \in S^{1}$ tal que que $\operatorname{Re}\left(\alpha \lambda_{1}\right)<0$ y $\operatorname{Re}\left(\alpha \lambda_{2}\right)<0$, dado $z=\left(z_{1}, z_{2}\right) \in \Gamma_{1}$, sabemos que $\mathcal{O}_{\alpha A}(z)=\left\{\varphi_{A}^{\alpha}(t, z) ; t \in \mathbb{R}\right\}$ corta transversalmente a $S^{3}$ en un único punto, es decir, existe un único $s_{0}=s_{0}(z) \in \mathbb{R}$ tal que $\varphi_{A}\left(s_{0}(z) \alpha, z\right) \in X$.

De esta manera, obtenemos la función $s_{0}: \Gamma_{1} \rightarrow \mathbb{R}$ definida implícitamente por la expresión

$$
\varphi_{A}\left(s_{0}(z) \alpha, z\right) \in X \text {. }
$$

Como esta $s_{0}$ es la retricción a $\Gamma_{1}$ de la función $s: U \rightarrow \mathbb{R}$ estudiada líneas arriba (la cual ya sabemos que es de clase $C^{\infty}$ ), se sigue que $s_{0}: \Gamma_{1} \rightarrow \mathbb{R}$ es de clase $C^{\infty}$.

Definimos $H: \Gamma_{1} \rightarrow X$ como

$$
H(z)=\varphi_{A}\left(s_{0}(z) \alpha, z\right)
$$

Claramente $H$ es una función de clase $C^{\infty}$.

Recíprocamente, podemos definir la función $G: X \rightarrow \Gamma_{1}$ como

$$
G(w)=\varphi_{A}\left(t_{0}(w) \alpha, w\right)
$$

donde $t_{0}(w) \in \mathbb{R}$ es el único tiempo real tal que la hoja $\mathcal{O}_{\alpha A}(w)$ corta a $\Gamma_{1}$. Se sigue que $G$ es de clase $C^{\infty}$ y que $G=H^{-1}$. De esta manera $L \cap S^{3}=H\left(\mathcal{O}_{\hat{\lambda}_{1} A}(z)\right)$ donde $z \in \Gamma_{1}$. Más específicamente, fijemos $z \in \Gamma_{1}$ y sea $L \in \mathcal{F}_{A}-\left\{\{(0,0)\},\{0\} \times \mathbb{C}^{*}\right\}$ la hoja que pasa por $z$. Si denotamos $\mu: \mathbb{R} \rightarrow \Gamma_{1}$ a la función $\mu(t)=\varphi_{A}^{\widehat{\lambda}_{1}}(t, z)$, sabemos que $L \cap \Gamma_{1}$ es el trazo de $\mu$, luego $L \cap S^{3}$ es el trazo de $\nu(t)=H(\mu(t))=\varphi_{A}\left(s_{0}(\mu(t)) \alpha, \mu(t)\right)$ y por tanto $\nu$ es suavemente difeomorfa a $\mu$.

Se sigue que $L \in \mathcal{F}_{A}-\left\{\{(0,0)\},\{0\} \times \mathbb{C}^{*}\right\}$ es topológicamente un cono con vértice en $(0,0)$ y directriz la curva $\nu$. 


\section{Estudio geométrico de las órbitas en el dominio de Siegel}

Sea $A=\left[\begin{array}{cc}\lambda_{1} & 0 \\ 0 & \lambda_{2}\end{array}\right] \in \mathbb{C}^{2 \times 2}$ con $\left(\lambda_{1}, \lambda_{2}\right) \in \mathcal{D}_{S}$. Como $\lambda_{2} / \lambda_{1} \in \mathbb{R}^{-}$entonces existen dos posibilidades: que el cociente sea racional negativo o que sea irracional negativo. Por el Teorema 2.2 de ocurrir la primera posibilidad, la curva $\mu: \mathbb{R} \rightarrow \mathbb{C}^{2}$ definida por $\mu(t)=\varphi_{A}^{\widehat{\lambda}_{1}}(t, z)$ es una curva cerrada en el toro y si ocurre la segunda posibilidad $\mu$ es una curva densa. De manera completamente análoga a lo realizado en el dominio de Poincaré (ver los Lemas (4.1) y (4.2)), se puede demostrar el siguiente resultado:

Lema 5.1. Sea $A=\left[\begin{array}{cc}\lambda_{1} & 0 \\ 0 & \lambda_{2}\end{array}\right] \in \mathbb{C}^{2 \times 2} \operatorname{con}\left(\lambda_{1}, \lambda_{2}\right) \in \mathcal{D}_{S}$. Si $L \in \mathcal{F}_{A}-\left\{\{(0,0)\},\{0\} \times \mathbb{C}^{*}\right\}$ entonces $L$ es transversal al cilindro $\Gamma_{1}$, más aún

$$
L \cap \Gamma_{1}=\left\{\varphi_{A}^{\hat{\lambda}_{1}}(t, z) ; t \in \mathbb{R}\right\}
$$

donde $z$ es cualquier punto de $L \cap \Gamma_{1}$.

Observación: A diferencia de lo que ocurría en el caso del dominio de Poincaré, ahora las hojas de $\mathcal{F}_{A}$ no interceptan transversalmente a las esferas $S_{r}^{3}$. La demostración queda como ejercicio para el lector.

Para determinar una segunda dirección linealmente independiente con $\hat{\lambda}_{1}$, por la Proposición 3.3, sabemos que existe $\alpha \in \mathbb{C}-\{0\}$ tal que $\alpha \lambda_{1}, \alpha \lambda_{2} \in \mathbb{R}$ y $\left(\alpha \lambda_{1}\right)\left(\alpha \lambda_{2}\right)<0$. Máss aún, podemos tomar $\alpha=\lambda_{1}^{-1}$ (el cual es claramente ortogonal y por tanto $\mathbb{R}$-linealmente independiente con $\hat{\lambda}_{1}$ ) y considerar el flujo real $\varphi_{A}^{\lambda_{1}^{-1}}: \mathbb{R} \times \mathbb{C}^{2} \rightarrow \mathbb{C}^{2}$ inducido por $\varphi$, es decir

$$
\varphi_{A}^{\lambda_{1}^{-1}}\left(t, z_{1}, z_{2}\right)=\left(e^{t} z_{1}, e^{t \lambda_{2} / \lambda_{1}} z_{2}\right)
$$

Denotando $c=\lambda_{2} / \lambda_{1} \in \mathbb{R}^{-}$, la matriz compleja $\lambda_{1}^{-1} A=\left[\begin{array}{cc}1 & 0 \\ 0 & \lambda_{2} / \lambda_{1}\end{array}\right] \in \mathbb{C}^{2 \times 2}$ puede ser identificada con la matriz real

$$
\lambda_{1}^{-1} A=\left[\begin{array}{cccc}
1 & 0 & 0 & 0 \\
0 & 1 & 0 & 0 \\
0 & 0 & c & 0 \\
0 & 0 & 0 & c
\end{array}\right] \in \mathbb{R}^{4 \times 4}
$$

Se sigue que $\lambda_{1}^{-1} A$ es una matriz hiperbólica (real) de índice 2 , y por tanto el origen de $\mathbb{C}^{2}=\mathbb{R}^{4}$ es una silla en donde el subespacio inestable puede ser identificado con $\mathbb{C} \times\{0\}$ y el subespacio estable con $\{0\} \times \mathbb{C}$. Más aún, el flujo real $\varphi_{\lambda_{1}^{-1} A}: \mathbb{R} \times \mathbb{R}^{4} \rightarrow \mathbb{R}^{4}$ puede ser identificado con el flujo real $\varphi_{A}^{\lambda_{1}^{-1}}: \mathbb{R} \times \mathbb{C}^{2} \rightarrow \mathbb{C}^{2}$ inducido por $\varphi_{A}$ en la dirección $\lambda_{1}^{-1}$.

Resumimos nuestros resultados en el siguiente teorema.

Teorema 5.1. Si $A=\left[\begin{array}{cc}\lambda_{1} & 0 \\ 0 & \lambda_{2}\end{array}\right] \in \mathbb{C}^{2 \times 2}$ con $\left(\lambda_{1}, \lambda_{2}\right) \in \mathcal{D}_{S}$ entonces

1. Existe un flujo real (lineal) cuyas hojas están contenidas en las hojas de $\mathcal{F}_{A}$ y para el cual $(0,0) \in \mathbb{C}^{2}$ es una silla con subespacio estable $\{0\} \times \mathbb{C}$ y subespacio inestable $\mathbb{C} \times\{0\}$. 
2. Las hojas de $\mathcal{F}_{A}-\{(0,0),\{0\} \times \mathbb{C}\}$ son transversales al cilindro $\Gamma_{1}=S^{1} \times \mathbb{C}$ y la curva real obtenida al intersectar cualquiera de estas hojas con $\Gamma_{1}$ están parametrizadas por el flujo real inducido $\varphi_{A}^{\hat{\lambda}_{1}}$.

De lo anterior, se puede inferir que toda hoja de $\mathcal{F}_{A}-\left\{(0,0), \mathbb{C}^{*} \times\{0\},\{0\} \times \mathbb{C}^{*}\right\}$ tiene una distancia positiva al origen. Vamos a demostrar este aserto. Consideremos la función $f: \mathbb{C}^{2} \rightarrow \mathbb{R}$ definida por

$$
f(z)=\|z\|^{2}=\langle z, z\rangle_{\mathbb{C}}
$$

Geométricamente, $f(z)$ es el cuadrado de la distancia del punto $z$ al origen.

Sea $L \in \mathcal{F}_{A}-\left\{(0,0), \mathbb{C}^{*} \times\{0\},\{0\} \times \mathbb{C}^{*}\right\}$, vamos a minimizar la restricción de $f$ a $L$. Si identificamos $\mathbb{C}^{2}$ con $\mathbb{R}^{4}$ y observamos $L$ como una superficie suave real de dimensión 2 , entonces podemos hallar los puntos críticos de $\left.f\right|_{L}$.

Recordemos que $z \in L$ es punto crítico de $\left.f\right|_{L}$ si y sólo si

$$
\langle\nabla f(z), v\rangle_{\mathbb{R}}=0, \quad \forall v \in T_{z} L=\{c A z ; c \in \mathbb{C}\}
$$

Tomando $c=1$, tenemos

$$
\langle\nabla f(z), A z\rangle_{\mathbb{R}}=\operatorname{Re}\left(\langle 2 z, A z\rangle_{\mathbb{C}}\right)=\operatorname{Re}\left(2 \bar{\lambda}_{1}\left|z_{1}\right|^{2}+2 \bar{\lambda}_{2}\left|z_{2}\right|^{2}\right)
$$

Tomando $c=i$, tenemos

$$
\begin{aligned}
\langle\nabla f(z), i A z\rangle_{\mathbb{R}} & =\operatorname{Re}\left(\langle 2 z, i A z\rangle_{\mathbb{C}}\right)=\operatorname{Re}\left(-2 i \bar{\lambda}_{1}\left|z_{1}\right|^{2}-2 i \bar{\lambda}_{2}\left|z_{2}\right|^{2}\right)=-\operatorname{Re}\left(\left[2 \bar{\lambda}_{1}\left|z_{1}\right|^{2}+2 \bar{\lambda}_{2}\left|z_{2}\right|^{2}\right] i\right) \\
& =\operatorname{Im}\left(2 \bar{\lambda}_{1}\left|z_{1}\right|^{2}+2 \bar{\lambda}_{2}\left|z_{2}\right|^{2}\right)
\end{aligned}
$$

De (6) y (7) concluímos que $z \in L$ es punto crítico de $\left.f\right|_{L}$ si y sólo si

$$
\operatorname{Re}\left(2 \bar{\lambda}_{1}\left|z_{1}\right|^{2}+2 \bar{\lambda}_{2}\left|z_{2}\right|^{2}\right)=0 \quad \text { y } \quad \operatorname{Im}\left(2 \bar{\lambda}_{1}\left|z_{1}\right|^{2}+2 \bar{\lambda}_{2}\left|z_{2}\right|^{2}\right)=0
$$

si y sólo si

$$
\lambda_{1}\left|z_{1}\right|^{2}+\lambda_{2}\left|z_{2}\right|^{2}=0
$$

Teniendo en cuenta que $\left(\lambda_{1}, \lambda_{2}\right) \in D_{S}$, entonces

$$
M=\left\{\left(z_{1}, z_{2}\right) \in \mathbb{C}^{2}-\{(0,0)\} ; \lambda_{1}\left|z_{1}\right|^{2}+\lambda_{2}\left|z_{2}\right|^{2}=0\right\}
$$

es una superficie suave (real) de codimensión 1. Además, no es difícil probar que $M$ intersecta transversalmente a la hoja $L$. Como el conjunto de los puntos críticos de $\left.f\right|_{L}$ viene dado por

$$
M \cap L=\left\{\left(z_{1}, z_{2}\right) \in L ; \lambda_{1}\left|z_{1}\right|^{2}+\lambda_{2}\left|z_{2}\right|^{2}=0\right\}
$$

concluímos que el conjunto de los puntos críticos de $\left.f\right|_{L}$ es una curva suave (unidimensional real) contenida en la hoja $L$. Usando la parametrización de $L$, vamos a demostrar que es posible parametrizar $L \cap M$. En efecto, sea $z_{0}=\left(z_{1}^{0}, z_{2}^{0}\right) \in \mathbb{C}^{*} \times \mathbb{C}^{*}$ tal que $L=\mathcal{O}\left(z_{0}\right)$. Entonces $L$ queda parametrizada por

$$
z(T)=\left(z_{1}(T), z_{2}(T)\right)=\left(e^{\lambda_{1} T} z_{1}^{0}, e^{\lambda_{2} T} z_{2}^{0}\right), \quad \forall T \in \mathbb{C}
$$


Se tiene entonces que

$$
\begin{aligned}
z(T) \in M & \Longleftrightarrow-\frac{\lambda_{2}}{\lambda_{1}}=\frac{\left|z_{1}(T)\right|^{2}}{\left|z_{1}(T)\right|^{2}}=e^{2 \operatorname{Re}\left[\left(\lambda_{2}-\lambda_{1}\right) T\right]} \frac{\left|z_{1}^{0}\right|^{2}}{\left|z_{1}^{0}\right|^{2}} \Longleftrightarrow e^{2 \operatorname{Re}\left[\left(\lambda_{2}-\lambda_{1}\right) T\right]}=\frac{\lambda_{2}\left|z_{1}^{0}\right|^{2}}{\lambda_{1}\left|z_{1}^{0}\right|^{2}} \\
& \Longleftrightarrow \operatorname{Re}\left[\left(\lambda_{2}-\lambda_{1}\right) T\right]=\ln \left(\sqrt{-\frac{\lambda_{2}}{\lambda_{1}}} \frac{\left|z_{1}^{0}\right|}{\left|z_{1}^{0}\right|}\right)=c \Longleftrightarrow\left(\lambda_{2}-\lambda_{1}\right) T=c+i s, \quad s \in \mathbb{R}
\end{aligned}
$$

Denotando $r=\frac{\lambda_{2}}{\lambda_{1}}$ se llega a

$$
z(T) \in M \Longleftrightarrow T=\frac{c+i s}{(1-r) \lambda_{1}}=\frac{c}{1-r} \lambda_{1}^{-1}+t \hat{\lambda}_{1}=\nu(t), \quad t \in \mathbb{R}
$$

De esta manera $L \cap M$ queda parametrizado por la curva $\nu: \mathbb{R} \rightarrow L \cap M$.

Un fácil cálculo muestra que

$$
f(\nu(t))=e^{2(1-r)^{-1}}\left|z_{1}^{0}\right|^{2}+e^{2\left(r^{-1}-1\right)^{-1}}\left|z_{2}^{0}\right|^{2}>0, \quad \forall t \in \mathbb{R}
$$

Concluímos que todo punto de $L \cap M$ es un mínimo de $\left.f\right|_{L}$. Resumimos todo lo obtenido en el siguiente teorema.

Teorema 5.2. Sea $A=\left[\begin{array}{cc}\lambda_{1} & 0 \\ 0 & \lambda_{2}\end{array}\right] \in \mathbb{C}^{2 \times 2}$ con $\left(\lambda_{1}, \lambda_{2}\right) \in \mathcal{D}_{S}$. Si $L \in \mathcal{F}_{A}-$ $\left\{(0,0), \mathbb{C}^{*} \times\{0\},\{0\} \times \mathbb{C}^{*}\right\}$ entonces $L$ tiene una distancia positiva al origen, más aún el conjunto de puntos de $L$ que realizan la distancia viene dado por la curva suave real

$$
\left\{\left(z_{1}, z_{2}\right) \in L ; \lambda_{1}\left|z_{1}\right|^{2}+\lambda_{2}\left|z_{2}\right|^{2}=0\right\}
$$

Observación: Las hojas de $\mathcal{F}_{A}$ que tienen una distancia positiva al origen son llamadas hoja de Siegel. El Teorema anterior nos dice que si $A=\left[\begin{array}{cc}\lambda_{1} & 0 \\ 0 & \lambda_{2}\end{array}\right] \in \mathbb{C}^{2 \times 2}$ con $\left(\lambda_{1}, \lambda_{2}\right) \in \mathcal{D}_{S}$ entonces toda hoja de $\mathcal{F}_{A}$ (distinta del origen y de los ejes) es una hoja de Siegel.

Para finalizar, observamos que los Teoremas (2.2), (4.1) y (5.2) describen completamente el comportamiento geométrico de las soluciones de una EDO lineal asociada a una matriz inversible y diagonalizable. 


\section{REFERENCIAS BIBLIOGRÁFICAS}

[1] Benazic, R. (2008) Tópicos de dinámica compleja, Notas de clase, Lima - Perú.

[2] Benazic, R. (2007) Tópicos de Ecuaciones Diferenciales Ordinarias, UNI, Lima - Perú.

[3] Benazic, R. (1996) Singularidades de Campos Vectoriales Holomorfos en el Dominio de Poincaré, PRO MATHEMATICA, Vol. X Nos. 19-20, pp. 9 -33.

[4] Calvo, O. (1998) Sistemas ineales Complejos, Aportaciones Matemáticas, Comunicaciones 21, pp. $95-120$.

[5] Camacho, C. (1988) Lectures on Complex 2-Dimensional Dynamical Systems, ICTP, Trieste - Italy.

[6] Camacho, C. (1988) Lectures on Holomorphyc Dynamical Systems, ICTP, Trieste - Italy.

[7] Camacho, C. , Sad, P. (1987) Pontos Singulares de Equações Diferenciais Analiticas, $16^{\circ}$ Colóquio Brasileiro de Matemática. Rio de Janeiro - Brasil.

[8] Guillemin, V. - Pollack, A. (1974) Differential Topology, Prentice-Hall, Inc., USA.

[9] Lima, E. (1973) Variedades diferenciáveis, Monografias de Matemática No15. Brasil.

[10] Sotomayor, J. (1979) Lições de Equações Diferenciais Ordinárias, Projeto Euclides, IMPA, Rio de Janeiro - Brasil. 